POPULATIONS Populations vulnérables

VULNERABLES $7 \mid 2021$

Mobilité résidentielle et vulnérabilités

\title{
L'accession à la propriété après les séparations : pourquoi un tel écart entre hommes et femmes?
}

Homeownership after marital separation: why is there such a gap between men and women?

Laure Crepin

\section{OpenEdition}

Journals

Édition électronique

URL : https://journals.openedition.org/popvuln/780

DOI : $10.4000 /$ popvuln.780

ISSN : 2650-7684

Éditeur

LIR3S - Laboratoire Interdisciplinaire de Recherche "Sociétés Sensibilités Soin" (UMR 7366 CNRS-uB)

Référence électronique

Laure Crepin, «L'accession à la propriété après les séparations : pourquoi un tel écart entre hommes et femmes? », Populations vulnérables [En ligne], 7| 2021, mis en ligne le 01 décembre 2021, consulté le 26 février 2022. URL : http://journals.openedition.org/popvuln/780 ; DOI : https://doi.org/10.4000/ popvuln.780

Ce document a été généré automatiquement le 26 février 2022.

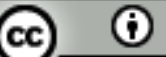

Les contenus de la revue Populations vulnérables sont mis à disposition selon les termes de la Licence Creative Commons Attribution 4.0 International 


\title{
L'accession à la propriété après les séparations : pourquoi un tel écart entre hommes et femmes?
}

\author{
Homeownership after marital separation: why is there such a gap between men \\ and women?
}

Laure Crepin

\section{Introduction}

En 2013, en France, près de $58 \%$ des ménages français sont propriétaires de leur logement (ENL 2013). Signe de stabilité conjugale et d'engagement familial (Bourdieu et Saint-Martin, 1990), les trois quarts de ces logements sont possédés en couple, le plus souvent à parts égales (Gollac, 2018). Lors des ruptures conjugales, la propriété du logement devient alors un enjeu de négociations, avec des conséquences résidentielles et patrimoniales importantes ${ }^{1}: 34 \%$ des propriétaires en couple perdent ce statut à l'occasion d'une séparation (ENL 2013). Or, les désunions constituent un phénomène fréquent. En France, chaque année, environ 425000 couples se séparent (Costemalle, 2017).

Dans la population globale, la perte du statut de propriétaire est un événement marginal. Seuls 3,8\% des propriétaires en 2009 ne le sont plus en 2013. Mais les séparations contribuent largement à ce phénomène : $41,9 \%$ des ménages ayant perdu récemment le statut de propriétaires l'ont quitté à la suite d'une séparation (ENL 2013). Or, devenir et rester propriétaire est crucial car la propriété a été construite, notamment par les politiques publiques depuis les années 1970 via l'encouragement aux crédits immobiliers, comme le statut d'occupation valorisé ${ }^{2}$ (Bourdieu, 2000 ; BugejaBloch, 2013). Elle constitue dans un contexte d'affaiblissement des mécanismes de protection sociale, d'individualisation et de "repatrimonialisation des modes de protection» (Chauvel, 2016), une assurance contre les nouveaux risques sociaux (vieillesse, précarisation de l'emploi, etc.) (Abdelnour et Lambert, 2014), et une 
protection statutaire pour les classes moyennes (Cusin, 2012). L'augmentation des inégalités s'explique d'ailleurs de plus en plus par l'inégale répartition du patrimoine (Piketty, 2013).

3 Or, après une séparation conjugale ${ }^{3}$, les femmes sont beaucoup moins propriétaires que les hommes en France (Durier, 2017 ; Ferrari et al., 2019), comme ailleurs en Europe (Feijten, 2005 ; Kulu et Mikulai, 2018 ; Andress et Hummelsheim, 2009). La séparation aggrave les inégalités de patrimoine entre hommes et femmes, inégalités déjà existantes dans la population générale (Gollac, 2017 ; Bonnet et al., 2014), et croissantes, malgré la baisse des inégalités professionnelles entre hommes et femmes (Frémeaux et Leturcq, 2018). Les successions et les séparations constituent dès lors deux moments particulièrement visibles de la construction de ces inégalités de genre et de leur renforcement (Bessière et Gollac, 2020).

Si la littérature sur la question a bien identifié l'existence d'inégalités genrées d'accès à la propriété au moment de la séparation, les mécanismes les générant sont moins connus. Le rôle de l'institution judiciaire (Collectif Onze, 2013) et des métiers du droit (Bessière et Gollac, 2020) a par exemple été mis en avant. Mais dans d'autres travaux (par exemple Ferrari et al., 2019), la propriété est souvent traitée de manière annexe via la question de la garde des enfants ou du logement conjugal. Or, par ses dimensions statutaires, patrimoniales, économiques, celle-ci participe à renforcer les inégalités entre les hommes et les femmes, ces dernières s'appauvrissant davantage en termes de revenus avec la séparation (Vanderschelden, 2014).

Nous identifions un double mouvement participant au renforcement d'inégalités genrées d'accession à la propriété lors de la séparation. D'une part, les trajectoires professionnelles genrées, souvent liées à des contraintes familiales avant la séparation, participent à expliquer les différences de propriété entre hommes et femmes. D'autre part, à conditions de vie similaires, les choix résidentiels diffèrent : les hommes sont plus enclins à devenir ou à rester propriétaires après une séparation.

Pour cela, nous exploitons les vagues de 2004 à $2014^{4}$ de l'enquête «Statistiques sur les ressources et les conditions de vie » $(\mathrm{SRCV})^{5}$, volet concernant la France métropolitaine de l'enquête européenne EU-SILC. Cette enquête individuelle et longitudinale permet de suivre la trajectoire de 940 individus séparés d'une union cohabitante, quel que soit son statut légal ${ }^{6}$, un an avant la séparation, et deux ans après. Ce laps de temps permet de comparer des situations résidentielles stabilisées. Nous exploitons occasionnellement l'Enquête nationale logement 2013 (ENL 2013), réalisée par l'INSEE, pour des statistiques complémentaires.

\section{Des inégalités d'accession à la propriété liées aux trajectoires professionnelles et familiales genrées}

\section{1) Des inégalités genrées d'accession à la propriété après une séparation}

7 Derrière la question de la propriété se cachent deux réalités différentes. Les propriétaires endettées, ou accédant·es à la propriété ( $20,1 \%$ des ménages français en 2013) remboursent des crédits immobiliers. L'accès ou non au crédit, ainsi que le taux d'intérêt dépendent des caractéristiques du ménage, du statut d'emploi et du montant 
des revenus, même si l'accès au crédit a été facilité au cours des années 2000 (Lambert, 2015). Les propriétaires non endettées, ou propriétaires non accédant-es $(37,7 \%$ des ménages), en moyenne plus âgées, possèdent pleinement leur bien.

Or, l'âge de la séparation est aussi l'âge de l'accession à la propriété endettée. Les séparations conjugales, tout comme les décisions d'achat immobilier, ont très majoritairement lieu entre 30 et 50 ans. Il est donc logique que de nombreux.ses séparées soient propriétaires endettées avant la séparation : c'est le cas de $34,5 \%$ des séparées, quand $13,5 \%$ sont propriétaires non endettées ${ }^{7}$. Cependant, les trajectoires des propriétaires endettées et non endettées diffèrent après une séparation.

Figure 1. Trajectoires des propriétaires endetté·es et non endetté·es en fonction du sexe

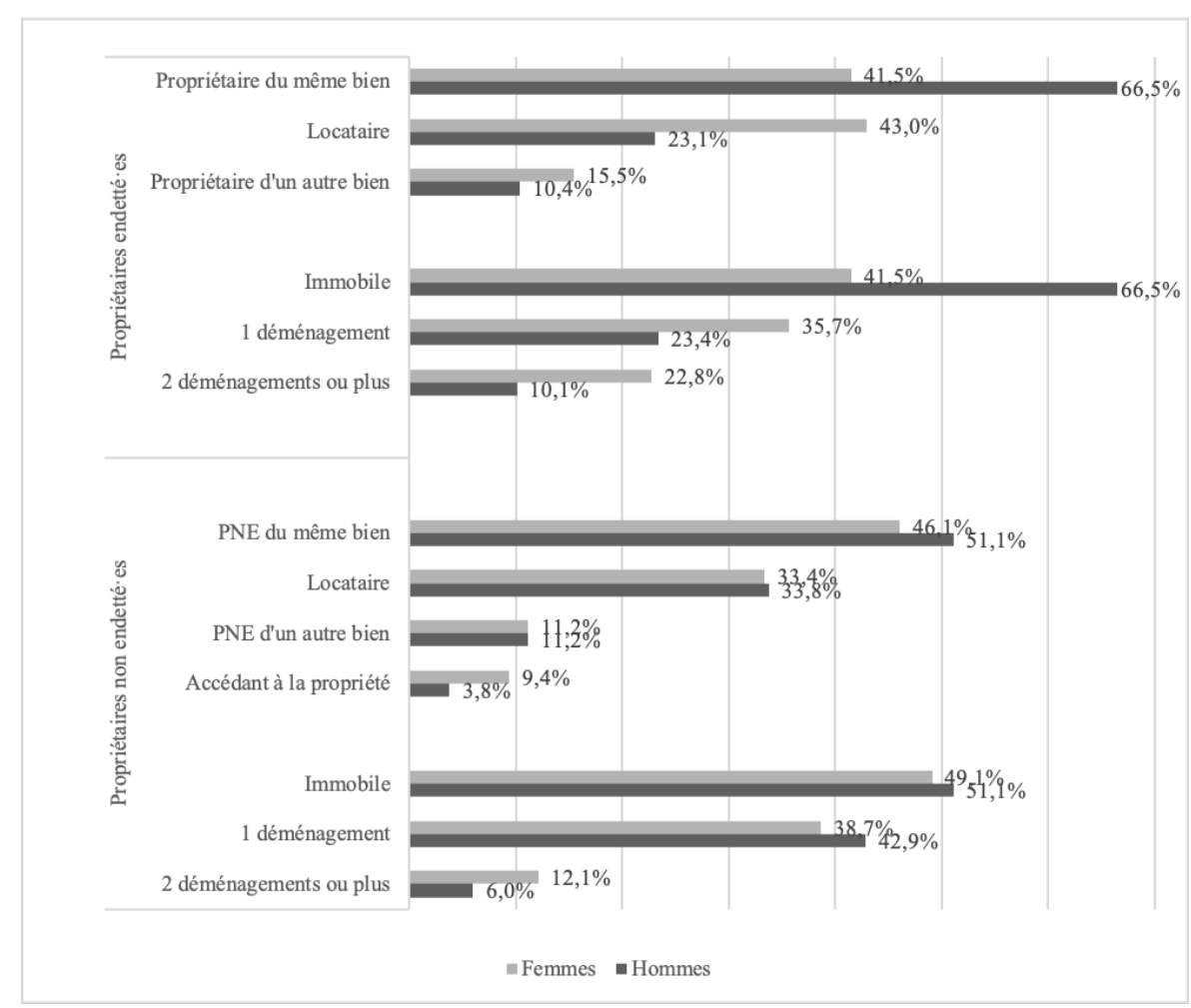

Champ : les propriétaires avant la séparation

Note de lecture : 66,5\% des hommes propriétaires endettés avant la séparation sont propriétaires de l'ancien logement conjugal après la séparation

PNE signifie propriétaire non endetté:e

Source : Enquête nationale logement 2013 (INSEE) 8

9 Pour les propriétaires non endettées en couple, il n'existe pas de différence significative entre les trajectoires résidentielles des hommes et des femmes après une rupture. En revanche, concernant les propriétaires endettées avant la séparation, les trajectoires des hommes et des femmes sont significativement différentes : les femmes déménagent plus, et restent moins propriétaires. Elles sont aussi plus fréquemment propriétaires d'un autre bien après la séparation, du fait de leur plus grande mobilité. C'est pourquoi nous nous intéressons aux trajectoires d'accession à la propriété (c'està-dire de propriétaires endettées) dans le cadre de cet article9.

La présence d'un crédit immobilier aggrave en effet les inégalités de genre, alors que la propriété non endettée permet les arrangements entre ex-conjoint-es sans intervention des banques, avec généralement un patrimoine plus important puisque le bien est 
remboursé. Ces inégalités d'accès au crédit immobilier s'appuient sur des inégalités dans la sphère domestique et professionnelle, qui s'accentuent avec l'entrée dans la parentalité.

\section{2) Des difficultés professionnelles liées aux arbitrages conjugaux pour les femmes}

11 Les couples propriétaires endettés avant la séparation arbitrent avec l'arrivée d'enfants en faveur de la baisse de l'activité des femmes, celles-ci étant pénalisées sur le marché du travail, malgré leur niveau de diplôme (Meurs et al., 2011).

Le tableau ci-dessous décrit les configurations professionnelles (Pochic, 2003) de couples propriétaires endettés avant leur séparation. Elles illustrent la place des hommes et des femmes dans les arbitrages professionnels réalisés par les couples.

Tableau 1. Configuration professionnelle des couples propriétaires endettés (\% en colonnes)

\begin{tabular}{|c|c|c|c|}
\hline & Femmes & Hommes & Ensemble \\
\hline \multicolumn{4}{|l|}{ Situation professionnelle } \\
\hline Deux actif·ves occupées & 82,2 & 78,6 & 80,6 \\
\hline En emploi avec conjoint $\cdot e$ au chômage ou inactif.ve & 3,1 & 15 & 8,7 \\
\hline Inactif ou chômage avec conjointe en emploi & 14,7 & 6,9 & 11,2 \\
\hline Total & 100 & 100 & 100 \\
\hline \multicolumn{4}{|l|}{ Professions et catégories socioprofessionnelles (PCS) } \\
\hline Cadres avec cadres & 7,5 & 6,5 & 7,1 \\
\hline Professions intermédiaires et petit·es indépendant·es ensemble & 24,3 & 15,4 & 20,4 \\
\hline Ouvrier-ères et Employé'es ensemble & 31,6 & 31,0 & 31,3 \\
\hline Conjoint $\cdot e$ avec une PCS inférieure ${ }^{10}$ & 14,6 & 34,6 & 23,2 \\
\hline Conjoint $\cdot$ e avec une PCS supérieure & 22,1 & 12,6 & 18,0 \\
\hline Total & 100 & 100 & 100 \\
\hline
\end{tabular}

Champ : les couples propriétaires endettés avant la séparation ( $N=312)$

Note de lecture : 22,1\% des femmes propriétaires endettées en couple avaient une catégorie socioprofessionnelle de statut inférieur à celle de leur conjoint

Source : SRCV (2004-2015)

13 Ce tableau souligne les dépendances économiques des femmes envers les hommes au sein des couples propriétaires endettés : dans $15 \%$ des cas, ces couples sont composés de femmes inactives ou au chômage, alors que les hommes sont en emploi. La réciproque est beaucoup moins fréquente. Les femmes sont également bien plus 
fréquemment en couple hypergame qu'hypogame, contrairement aux hommes : 22,1\% des femmes propriétaires endettées en couple vivent avec un conjoint dont la PCS est supérieure, contre $12,6 \%$ des hommes ${ }^{11}$. Comment l'expliquer ? Les couples accédants à la propriété sont plus souvent mariés (Sarna, 1985) et plus fréquemment parents que les autres couples ( $82 \%$ des couples accédants à la propriété ont des enfants au moment de la séparation). Or, ces signes d'engagement familial incitent à la spécialisation domestique des femmes (Meurs et al., 2011) : c'est à l'arrivée des enfants que l'écart salarial entre hommes et femmes augmente du fait de discriminations dans la sphère professionnelle et d'une baisse de l'activité, les femmes s'engageant toujours plus que les hommes dans le travail parental quotidien (Blöss, 2016). Au contraire, la présence d'enfant a un effet bénéfique sur la trajectoire professionnelle masculine : les hommes sont plus susceptibles de connaître des promotions avec la paternité (Andress et Hummelsheim, 2009). Pourtant, les femmes sont en moyenne plus diplômées que les hommes, mais ces diplômes sont moins convertis en professions valorisées (Vallot, 2019).

14 Avant la séparation, les femmes cumulent donc les difficultés professionnelles liées aux arbitrages effectués en couple. Au moment de la séparation, elles ont non seulement des situations professionnelles plus précaires, mais aussi en grande majorité la garde des enfants du couple ${ }^{12}$, comme le montre le tableau 2.

Tableau 2. Caractéristiques des propriétaires endettés avant la séparation deux ans après la séparation (\% en colonnes)

\begin{tabular}{|c|c|c|c|}
\hline & Femmes & Hommes & Ensemble \\
\hline \multicolumn{4}{|l|}{ Situation d'emploi deux ans après la séparation } \\
\hline CDI temps plein & 44,8 & 64,5 & 53,4 \\
\hline CDI temps partiel & 14,4 & 1,7 & 8,9 \\
\hline Indépendant, chef d'entreprise & 10,9 & 9,9 & 10,5 \\
\hline Chômeur & 5,9 & 8,9 & 7,2 \\
\hline Emploi aidé, CDD, intérim & 15,4 & 14,1 & 14,8 \\
\hline Inactif & 8,6 & 0,8 & 5,2 \\
\hline Total & 100 & 100 & 100 \\
\hline \multicolumn{4}{|l|}{ Type de ménage deux ans après la séparation } \\
\hline Couple avec enfant(s) & 17,5 & 15,2 & 16,5 \\
\hline Couple sans enfant(s) ou enfant(s) hors domicile & 6 & 7,9 & 6,9 \\
\hline Ménage monoparental & 57,2 & 27,2 & 44 \\
\hline Personne seule avec enfant(s) hors domicile & 7,5 & 25,1 & 15,2 \\
\hline
\end{tabular}




\begin{tabular}{|c|c|c|c|}
\hline Personne seule sans enfant(s) & 11,8 & 24,7 & 17,4 \\
\hline Total & 100 & 100 & 100 \\
\hline \multicolumn{4}{|l|}{ Niveau de vie deux ans après la séparation } \\
\hline $\begin{array}{l}\text { Niveau de vie après la séparation (euros/unité de consommation } \\
\text { et par mois) }\end{array}$ & 1680 & 1860 & 1760 \\
\hline Évolution du niveau de vie & $-13 \%$ & $-2 \%$ & $-8 \%$ \\
\hline
\end{tabular}

Champ : les propriétaires endetté es avant la séparation $(N=312)^{13}$

Note de lecture : $44,8 \%$ des femmes propriétaires endettées en couple occupent un emploi en CDI à temps plein deux ans après la séparation

Source : SRCV (2004 - 2015)

Après la séparation, les hommes occupent encore davantage que les femmes des emplois en CDI à temps plein. Pourtant, leur situation professionnelle se dégrade avec la rupture : avant celle-ci, 73,9\% des hommes ont un CDI à temps plein, contre $64,5 \%$ après la séparation. La désunion provoque effectivement une déstabilisation professionnelle pour certains hommes sans conjointe devant assurer la bonne marche de l'économie domestique (Andress et Hummelsheim, 2009). Inversement, les femmes retournent à l'emploi après cet événement. Néanmoins, elles ont souvent des enfants à charge, ce qui constitue un frein à la reprise du travail (Bonnet et al., 2009). L'année suivant la rupture conjugale, $71 \%$ des enfants sont gardés par leur mère, malgré une très forte progression de la garde alternée depuis les années $2000^{14}$ (Guillaunneau et Moreau, 2013). Le niveau de vie ${ }^{15}$ des femmes propriétaires endettées en couple baisse alors de $13 \%$, bien plus que celui des hommes, baisse du niveau de vie qui ne peut être compensée par le montant des pensions alimentaires versées aux mères, généralement insuffisant (Collectif Onze, 2013).

\section{3) Des caractéristiques féminines entraînant la perte du statut de propriétaire}

Les femmes ont des situations économiques défavorables par rapport aux hommes après la séparation. Plus souvent à la tête de familles monoparentales, elles sont moins en CDI à temps plein, et occupent moins souvent des positions de cadres que les hommes. Or, la figure 2 éclaire à quel point ces caractéristiques féminines sont corrélées à la perte du statut de propriétaire ${ }^{16}$. Les arbitrages professionnels et familiaux effectués pendant la mise en couple expliquent ainsi, en partie, les difficultés résidentielles des femmes après les séparations. 
Figure 2. Part des propriétaires endetté-es en couple perdant le statut de propriétaire après la séparation, en fonction de caractéristiques professionnelles

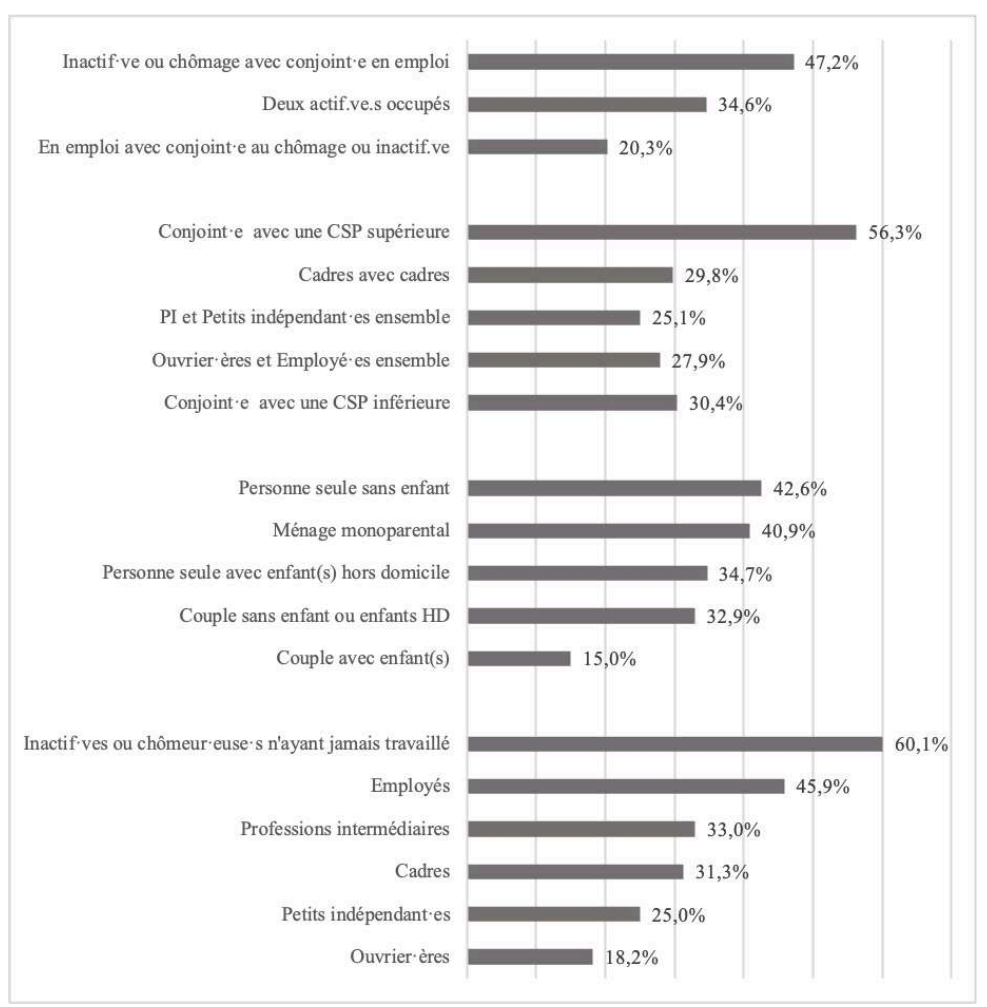

Champ : les propriétaires endetté.es avant la séparation $(\mathrm{N}=312)^{17}$

Note de lecture : 18,2\% des ouvrier.ères propriétaires endetté.es avant la séparation ont perdu le statut de propriétaire au moment de la séparation

Source : SRCV (2004-2015)

Les séparées en situation de dépendance économique (situation d'hypergamie, ou absence d'emploi contrairement à l'ex-conjointe) quittent plus souvent le statut de propriétaire. Par exemple, $47,2 \%$ des individus au chômage ou inactifs quand le conjointe a un emploi perdent ce statut au moment de la séparation, alors que c'est le cas de $20,3 \%$ des séparées dans la situation symétrique (en emploi, avec conjoint·e inactif·ve ou au chômage). De plus, la garde des enfants ne protège pas les propriétaires endettées en couple de la perte de leur statut résidentiel, bien au contraire : 40,9\% des ménages monoparentaux abandonnent le statut de propriétaire avec la séparation.

$\mathrm{Au}$ sein des classes populaires, les trajectoires résidentielles sont genrées à l'issue des désunions. $45,9 \%$ des employées (catégorie à $86,2 \%$ féminine), et $60,1 \%$ des inactif.ves propriétaires endettées en couple (93,6\% de femmes) n'ont plus le statut de propriétaire au moment de la rupture, contre $18,2 \%$ seulement des ouvrierères (population en grande majorité masculine: $78,2 \%$ d'hommes). Employées et ouvrierères ont pourtant des niveaux de vie d'environ 1500 euros/unité de consommation/mois. Peut-être est-ce lié au fait que les femmes employées sont plus fréquemment en couple hypergame que les hommes ouvriers. Les ouvriers bénéficient également davantage d'un CDI à temps plein après la séparation (70 \% des ouvriers), alors que c'est le cas de seulement $50 \%$ des employées. Enfin, ils bénéficient généralement de compétences techniques et de relations professionnelles leur permettant de rester propriétaires d'une maison à moindre coût, située dans une zone 
périphérique ${ }^{18}$, et dont ils tirent des avantages statutaires (Cartier et al., 2008 ; Lambert, 2015).

Ces contraintes professionnelles féminines contribuent grandement à expliquer l'écart de propriété entre hommes et femmes après une désunion, indépendamment des aspirations résidentielles éventuellement genrées à situation sociale équivalente. Pour estimer, toutes choses égales par ailleurs, l'influence des variables professionnelles sur la probabilité d'être propriétaire après une rupture conjugale, une régression a été réalisée (cf. Annexe). Celle-ci estime la probabilité d'être propriétaire deux ans après une séparation, en prenant en compte le sexe, mais aussi des variables professionnelles, résidentielles et familiales. Le contrôle par le sexe permet en effet d'éliminer le biais induit par les choix résidentiels genrés (cf. III). Les carrières moins rémunératrices, ainsi que les arbitrages en faveur de la carrière d'une des conjoint.es, influent sur la probabilité d'être propriétaire, à caractéristiques équivalentes. Les séparées en situation d'hypergamie ont 5 fois moins de chances d'être propriétaires après une désunion, toutes choses égales par ailleurs, par rapport aux couples où les deux conjointes sont cadres ou chefs d'entreprise. Être propriétaire après la séparation est également fortement lié au niveau de vie (cf. note 15). Les individus du premier quartile de niveau de vie ont 4,7 fois moins de chances, toutes choses égales par ailleurs, d'être propriétaire après une rupture, par rapport au dernier quartile. Or, le premier quartile est féminin à plus de $70 \%$. Ce sont donc les séparées dont la situation professionnelle est inférieure à celle de leur ex-conjoint·e, et qui ont les niveaux de vie les plus faibles post-rupture, qui ont le moins de chances d'être propriétaires, trajectoires professionnelles en grande majorité féminines.

Les résultats montrent la nécessité de lier les trajectoires familiales et professionnelles pour expliquer les inégalités genrées d'accès à la propriété après les séparations. L'entrelacement et l'accumulation de caractéristiques féminines désavantagent surtout un certain profil de femmes: les mères de ménage monoparental, celles de classes populaires, celles qui avaient pu accéder à la propriété grâce à la situation de leur conjoint. Toutefois, ce "cumul des inégalités » se double de choix genrés pour les hommes en faveur de la propriété.

\section{Des inégalités renforcées via des choix résidentiels genrés}

\section{1) Des choix résidentiels différents à niveau de vie similaire}

L'approche sociologique des "choix résidentiels " (Grafmeyer, 2010) étudie les marges de manœuvres individuelles, inégales en fonction des ressources sociales et des contraintes des marchés immobiliers et inscrites dans le temps long. La socialisation antérieure participe alors à façonner les préférences résidentielles au sein d'un univers des possibles plus ou moins contraint. Or, hommes et femmes n'ont pas les mêmes aspirations résidentielles. Dès l'après-guerre, des travaux montraient que les hommes valorisaient davantage la maison individuelle, en propriété, hors des grands centres urbains, alors que les femmes privilégiaient la centralité au statut de propriétaire (Bonvalet et INED, 2019). Ces clivages sont toujours d'actualité d'après la dernière Enquête Logement (2013). Les situations de séparations conjugales révèlent ces choix 
résidentiels genrés : à caractéristiques équivalentes, les hommes optent plus souvent après la séparation pour la propriété endettée que les femmes (Figure 3).

Figure 3. Taux de propriété endettée après la séparation des hommes et des femmes en fonction de caractéristiques professionnelles et économiques

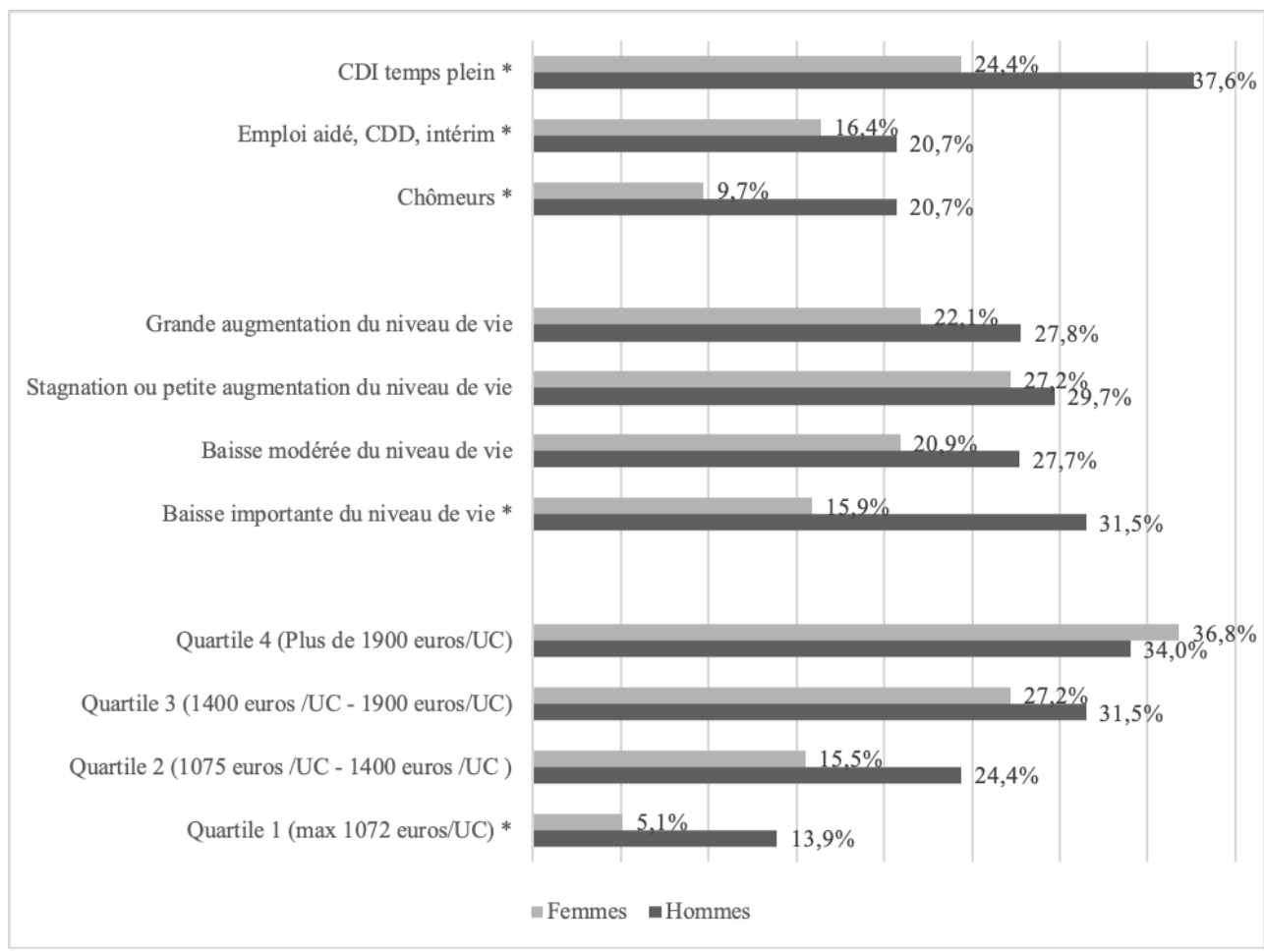

Champ : les séparé.e.s ( $\mathrm{N}=940)$

Note de lecture : 20,7 \% des hommes au chômage sont propriétaires endettés après la séparation Note : sont représentés ici (dans l'ordre) le statut de l'emploi deux ans après la séparation, les évolutions de niveau de vie et le quartile de niveau de vie deux ans après la séparation ${ }^{19}$ *différence significative entre hommes et femmes au seuil de $5 \%$

Source : SRCV (2004-2015)

On note une plus grande disparité entre hommes et femmes parmi les moins aisées et pour celles et ceux dont les revenus diminuent le plus : $31,5 \%$ des hommes qui ont vécu, au moment de la séparation, une contraction importante de leur niveau de vie sont propriétaires endettés, contre $15,9 \%$ des femmes dans la même situation. La propriété peut en effet compenser une position sociale fragilisée par une déstabilisation professionnelle ou une baisse de revenus (Vignal, 2013). À type d'emploi similaire, les femmes font aussi moins le choix de la propriété endettée que les hommes. Par exemple, 37,6 \% des hommes qui occupent un emploi en CDI à temps plein sont propriétaires endettés, contre $14,4 \%$ des femmes dans la même situation. C'est seulement parmi les plus aisés (le dernier quartile), que les hommes et les femmes sont propriétaires endettés dans les mêmes proportions.

Les écarts d'accession à la propriété entre les hommes et les femmes concernent donc surtout les classes populaires, ou moyenne, les individus dont les revenus sont les plus faibles, et celles et ceux pour lesquels le revenu baisse le plus du fait de la séparation ${ }^{20}$, et non les hommes et femmes les plus aisées. Si les contraintes professionnelles et financières expliquent, en partie, la moindre propension des femmes à être 
propriétaires, ainsi que les préférences genrées pour ce statut, d'autres caractéristiques viennent accentuer ces disparités.

\section{2) Des différences genrées accrues par la présence d'enfants}

Si les hommes sont plus propriétaires que les femmes, cette situation se renforce encore avec la présence d'enfants. Cela concerne surtout les moins de $50 \mathrm{ans}^{21}$. À partir de cet âge, la plupart des enfants ont quitté le domicile parental : $71 \%$ des séparés de 50 à 60 ans ont des enfants vivant hors du domicile, contre $35,4 \%$ des $40-50$ ans. Le tableau 3 se concentre donc sur les moins de 50 ans ayant des enfants, pour que la configuration d'enfants «hors domicile ${ }^{22}$ concerne les questions de garde après les séparations (et non des enfants étudiantes ou jeunes actif-ves) ${ }^{23}$. Cette population a en moyenne 2,04 enfants et compte $57,9 \%$ de ménages monoparentaux ${ }^{24}, 15,6 \%$ de personnes seules ayant des enfants hors domicile, le reste se composant de personnes remises en couples.

Tableau 3. Taux de propriété des hommes et des femmes deux ans après une séparation en fonction de caractéristiques familiales

\begin{tabular}{|c|c|c|c|c|c|}
\hline & \multicolumn{2}{|c|}{$\begin{array}{l}\text { Ménage } \\
\text { monoparental } \\
\text { de } 50 \text { ans) }\end{array}$} & \multirow{2}{*}{\begin{tabular}{|l} 
Seule avec enfants \\
Hors domicile (- \\
de 50 ans) \\
$\mathrm{H}^{26}$
\end{tabular}} & \multicolumn{2}{|c|}{$\begin{array}{l}25-50 \text { ans sans } \\
\text { enfant }^{25}\end{array}$} \\
\hline & $\mathrm{H}$ & F & & $\mathrm{H}$ & F \\
\hline $\begin{array}{l}\text { Taux de propriétaires } \\
\text { endettées }\end{array}$ & $50,9 \%$ & $20,4 \%$ & $32,9 \%$ & $28,5 \%$ & $18,7 \%$ \\
\hline $\begin{array}{l}\text { Taux de propriété (endettée } \\
\text { et non endettée comprises) }\end{array}$ & $55,8 \%$ & $21,5 \%$ & $33,8 \%$ & $31,8 \%$ & $22,4 \%$ \\
\hline
\end{tabular}

Champ : deux premières colonnes : les séparé es de moins de 50 ans au moment de la séparation, ayant un ou des enfants ( $N=513 ; 54,4 \%$ de la population des séparées). Troisième colonne : les 25-50 ans sans enfant $(\mathrm{N}=189)$

Note de lecture : 50,9\% des hommes à la tête de ménages monoparentaux sont propriétaires endettés

Source : SRCV (2004-2015)

Les femmes à la tête de ménages monoparentaux sont souvent locataires privées $(40,4 \%)$ et locataires sociales $(30,7 \%)$. Ce n'est pas le cas des pères, fréquemment propriétaires endettés, surtout lorsqu'ils vivent avec leurs enfants. L'acquisition d'un pavillon est généralement une prérogative masculine, notamment dans les milieux populaires (Lambert, 2015). Signe d'engagement familial au sein du couple et de respectabilité, les pères semblent poursuivre ce choix après la séparation.

Cependant, les hommes qui demandent la garde sont majoritairement issus de classes moyennes ou supérieures (Collectif Onze, 2013). Ils ont un niveau de vie d'environ 1500 euros par mois, contre 1300 euros par mois pour les mères vivant seules. Pour vérifier si les différences entre les hommes et les femmes sont dues à des effets de structure, ou à des arbitrages opposés toutes choses égales par ailleurs, nous utilisons la régression logistique présentée en annexe. Celle-ci montre que les variables professionnelles et de 
situation économique ne permettent pas d'expliquer entièrement les inégalités de propriété entre hommes et femmes ayant la garde des enfants, ni entre les hommes ayant la garde des enfants et ceux ayant des enfants hors domicile. Les femmes avec des enfants dans le domicile ont 2,9 fois moins de chances que les hommes dans la même situation d'être propriétaire après une séparation. De plus, avoir la garde des enfants contribue à la définition des projets résidentiels masculins: les hommes ayant des enfants hors domicile ont près de trois fois moins de chances d'être propriétaires par rapport aux hommes qui ont la garde des enfants, toutes choses égales par ailleurs.

On peut expliquer ces différences par les injonctions différenciées pesant sur les pères et les mères après une séparation. L'institution judiciaire participe à définir des modèles de paternité, et de maternité, qui influencent les trajectoires résidentielles et l'accumulation de patrimoine (Collectif Onze, 201327). Les injonctions maternelles au travail domestique ordinaire (Collectif Onze, 2013), déjà présentes avant la séparation malgré le principe de coparentalité (Blöss, 2016), sont alors réaffirmées. Or, cet investissement peut entraîner le désinvestissement, contraint ou choisi, d'autres sphères, professionnelles et conjugales, pour les mères. Pour les pères, au contraire, une situation économique prospère ainsi que la possibilité de transmettre un patrimoine sont valorisées (Orain, 2013). En outre, la garde des enfants encourage la sédentarité après les séparations, notamment pour les hommes (Martin, 1997). Ce double avantage (être un homme et avoir obtenu la garde des enfants) favorise la conservation du statut de propriétaire.

\section{Conclusion}

Au moment de la rupture, pourquoi les femmes perdent-elles bien plus fréquemment le statut de propriétaire, d'autant plus celui de propriétaire endettée alors qu'elles ont plus fréquemment la garde des enfants à l'issue de la désunion?

Tout d'abord, les femmes restent moins propriétaires parce qu'elles disposent de ressources plus faibles. Il existe des différences professionnelles entre hommes et femmes en partie créées par les arbitrages conjugaux lors de l'arrivée d'enfants. Ces arbitrages s'appuient et se justifient par les discriminations que subissent les femmes sur le marché de l'emploi, notamment lorsqu'elles ont des enfants (Meurs et al., 2011), et par des choix d'études moins valorisées, en anticipant leurs rôles familiaux (DuruBellat, 2003). Avant comme après la séparation, les femmes ont ainsi des carrières plus hachées, des positions professionnelles inférieures et de fait des niveaux de vie plus faibles, d'autant plus qu'elles ont plus souvent la garde des enfants du couple. Or, toutes ces caractéristiques sont associées à la perte du statut de propriétaire au moment de la séparation pour les propriétaires endettées en couple.

Cependant, l'accumulation de ces difficultés féminines ne permet d'expliquer qu'une partie des trajectoires résidentielles de femmes après les séparations, les hommes, à caractéristiques équivalentes, étant toujours davantage propriétaires, en particulier en bas de l'échelle sociale et lorsqu'ils ont la garde de leur enfant. Ces résultats mettent en évidence des injonctions sociales dessinant le portrait d'une paternité définie par la transmission de ressources patrimoniales. Au contraire, les mères, même en garde alternée, continuent à avoir prioritairement la charge éducative et de santé des enfants, engageant plus de dépenses, et notamment plus de dépenses courantes par rapport aux cadeaux exceptionnels (Cadolle, 2008). 
31 les moyens de rester propriétaires. Mais ces normes créent aussi des systèmes de préférence résidentielles genrés, qui participent à expliquer l'augmentation des inégalités de patrimoine entre hommes et femmes (Frémeaux et Leturcq, 2018). Or « la propriété individuelle semble progressivement remplacer la propriété sociale" (Abdelnour et Lambert, 2014) : l'accès à la propriété pallie le retrait de l'État dans la protection contre les risques sociaux (maladie, vieillesse, etc.). Ne plus être propriétaire constitue donc une fragilité supplémentaire. Ces inégalités sont particulièrement cruciales et impliquent de s'intéresser aux principaux mécanismes redistributeurs (la famille, l'État et l'emploi) et à leurs rôles dans la résorption ou l'aggravation de ces inégalités (Dewilde, 2008).

\section{BIBLIOGRAPHIE}

Abdelnour S. et Lambert A. (2014), « “L'entreprise de soi”, un nouveau mode de gestion politique des classes populaires. Analyse croisée de l'accession à la propriété et de l'auto-emploi (1977-2012) ", Genèses, vol. 95, n² 2, p. 27-48.

Amossé T. (2019), «Quelle définition statistique des classes populaires ? Propositions d'agrégation des situations socioprofessionnelles des ménages ", Sociétés contemporaines, vol. $114, \mathrm{n}^{\circ} 2$, p. 23-57.

Andress H.-J. et Hummelsheim D. (2009), When marriage ends: economic and social consequences of partnership dissolution, Cheltenham et Northampton, Edward Elgar Publishing, $432 \mathrm{p}$.

Bessière C. et Gollac S. (2020), Le genre du capital. Comment la famille reproduit les inégalités, Paris, La Découverte.

Blöss T. (2016), « Devoirs maternels, reproduction sociale et inégalités sexuées », Actes de la recherche en sciences sociales, vol. $214, \mathrm{n}^{\circ} 4$, p. 46-65.

Bonnet C., Solaz A. et Algava E. (2009), « La séparation conjugale affecte-t-elle l'activité professionnelle? Une estimation basée sur les méthodes d'appariement », Documents de travail, $\mathrm{n}^{\circ}$ 164, Paris, Ined.

Bonnet C., Keogh A. et Rapoport B. (2014), « Quels facteurs pour expliquer les écarts de patrimoine entre hommes et femmes en France? ", Économie et Statistique, vol. 491, n 1 , p. 101-123.

Bonvalet C. et Ined (2019), Désirs des Français en matière d'habitation urbaine : une enquête par sondage de 1945, Paris, Ined, $124 \mathrm{p}$.

Bourdieu P. et Saint Martin M. (de) (1990), « Le sens de la propriété », Actes de la recherche en sciences sociales, $\mathrm{n}^{\text {os }} 81-82$, p. 52-64.

Bourdieu P. (2000), Les structures sociales de l'économie, Paris, Seuil, 289 p.

Bugeja-Bloch F. (2013), Logement, la spirale des inégalités. Une nouvelle dimension de la fracture sociale et générationnelle, Paris, PUF, $272 \mathrm{p}$. 
Buisson G. et Lapinte A. (2013), « Le couple dans tous ses états. Non-cohabitation, conjoints de même sexe, Pacs... ", INSEE Première, n 1435.

Cadolle S. (2008), « La résidence alternée : ce qu'en disent les mères », Informations sociales, vol. $149, \mathrm{n}^{\circ} 1$, p. 1-4.

Cartier M. et al. (2008). La France des « petits-moyens », Paris, La Découverte, 309 p.

Chauvel L. (2016), La spirale du déclassement. Essai sur la société des illusions, Paris, Seuil, 224 p.

Collectif Onze (2013), Au tribunal des couples. Enquête sur des affaires familiales, Paris, Éditions Odile Jacob, 309 p.

Costemalle V. (2017), « Formations et ruptures d'unions : quelles sont les spécificités des unions libres? ", INSEE références - France portrait social, p. 91-110.

Cusin F. (2012), « Le logement, facteur de sécurisation pour des classes moyennes fragilisées? », Espaces et sociétés, vol. 148-149, n 1, p. 17-36.

Dewilde C. (2008), « Divorce and the Housing Movements of Owner-Occupiers: A European Comparison », Housing Studies, vol. 23, n 6, p. 809-832.

Durier S. (2017), « Après une rupture d'union, l'homme reste plus souvent dans le logement conjugal », INSEE Focus, vol. 91, p. 1-8.

Duru-Bellat M. (2003), L'école des filles. Quelle formation pour quels rôles sociaux?, Paris, L'Harmattan, $276 \mathrm{p}$.

Feijten P. (2005), « Union Dissolution, Unemployment and Moving Out of Homeownership », European Sociological Review, vol. 21, n 1, p. 59-71.

Ferrari G., Bonnet C. et Solaz A. (2019), «"Will the one who keeps the children keep the house?" Residential mobility after divorce by parenthood status and custody arrangements in France ", Demographic Research, vol. 40, n 14, p. 359-394.

Frémeaux N. et Leturcq M. (2018), « Prenuptial agreements and matrimonial property regimes in France, 1855-2010 », Explorations in Economic History, vol. 68, p. 132-142.

Gollac S. (2017), « Le genre caché de la propriété dans la France contemporaine », Cahiers du Genre, vol. 62, n 1, p. 43-59.

Gollac S. (2018), « Le patrimoine immobilier, une analyse du genre de la propriété », in Bonvalet C., Dietrich-Ragon P. et Lambert A. (dir), Le monde privé des femmes : genre et habitat dans la société française, Paris, Ined, p. 213-228.

Grafmeyer Y. (2010), « Chapitre 2 : approches sociologiques des choix résidentiels », in Authier J.-Y., Bonvalet C. et Lévy J.-P. (dir.), Élire domicile. La construction sociale des choix résidentiels, Lyon, Presses universitaires de Lyon, p. 58-75.

Guilleaunneau M. et Moreau C. (2013), «La résidence des enfants de parents séparés. De la demande des parents à la décision du juge ", rapport du ministère de la Justice, direction des affaires civiles et du sceau.

Kulu H. et Mikolai J. (2018), « Short- and Long-Term Effects of Divorce and Separation on Housing Tenure in England and Wales ", Population Studies, vol. 71, $\mathrm{n}^{\circ}$ 1, p. 17-39.

Lambert A. (2015), « Tous propriétaires! ». L'envers du décor pavillonnaire, Paris, Seuil, 288 p.

Martin C. (1997), L'Après divorce. Lien familial et vulnérabilité, Rennes, Presses universitaires de Rennes, $331 \mathrm{p}$. 
Meurs D., Pailhé A. et Ponthieux S. (2011), « Chapitre 6. Enfants, interruptions d'activité des femmes et écart de salaire entre les sexes ", in Milewski F. (dir.) Les discriminations entre les femmes et les hommes, Paris, Presses de Sciences Po, p. 139-162.

Orain R. (2013), « Focus - Paternité et enjeux professionnels », Informations sociales, vol. 176, $\mathrm{n}^{\circ} 2$, p. $58-60$.

Piketty T. (2013), Le capital au XXI e siècle, Paris, Seuil, 970 p.

Pochic S. (2003), « Le chômage des cadres : un révélateur des tensions entre carrière et vie privée? ", Actes de la journée du 20 juin 2003, IRESCO, p. 1-17.

Sarna C. (1985), « La cohabitation hors mariage : un mode de vie différent ? », Économie et statistique, vol. 179, p. 47-52.

Vallot P. (2019), « Le diplôme et la profession : deux composantes de la classe sociale en France et en Allemagne », L'Année sociologique, vol. 69, n² 2, p. 425-450.

Vanderschelden M. (2014), « Les ruptures familiales. État des lieux et propositions », rapport du Haut conseil de la famille.

Vignal C. (2013), « Ruptures du travail ouvrier et ruptures des rapports familiaux à la mobilité », Regards sociologiques, vol. 45-46, p. 206-217.

Volant S. (2017), « Un premier enfant à 28,5 ans en $2015: 4,5$ ans plus tard qu'en 1974 », INSEE Première, $\mathrm{n}^{\circ} 1642$.

\section{ANNEXES}

La régression logistique ci-après permet de modéliser la probabilité d'être propriétaire deux ans après une séparation indépendamment de variables professionnelles, résidentielles et familiales. Les variables du modèle permettent d'expliquer $52 \%$ de la variable réponse.

Tableau 4. Modèle de régression expliquant la probabilité d'être propriétaire après une séparation

\begin{tabular}{|c|c|c|c|}
\hline Variable et modalité de référence & Modalité active & $\begin{array}{l}\text { Odds } \\
\text { Ratio }\end{array}$ & P-value \\
\hline & Constante & & $0,02^{*}$ \\
\hline \multirow{5}{*}{$\begin{array}{l}\text { Situation familiale } \\
\text { Réf. : } \mathrm{H} \text { avec enfant(s) dans le domicile }\end{array}$} & $\begin{array}{l}\text { F. avec enfant(s) hors } \\
\text { domicile }\end{array}$ & 0,21 & $0,01^{*}$ \\
\hline & F. sans enfant & ns & 0,84 \\
\hline & $\begin{array}{l}\text { F. avec enfant(s) dans le } \\
\text { domicile }\end{array}$ & 0,35 & $0,02^{*}$ \\
\hline & $\begin{array}{l}\text { H. avec enfant(s) hors } \\
\text { domicile }\end{array}$ & 0,34 & $0,04^{*}$ \\
\hline & H. sans enfant & ns & 0,98 \\
\hline
\end{tabular}




\begin{tabular}{|c|c|c|c|}
\hline \multirow{2}{*}{$\begin{array}{l}\text { Situation conjugale } \\
\text { Réf. : célibataire }\end{array}$} & En couple cohabitant & 2,56 & $0,03 *$ \\
\hline & En couple non-cohabitant & ns & 0,82 \\
\hline \multirow{3}{*}{$\begin{array}{l}\text { Âge lors de la séparation } \\
\text { Réf. : } 30-40\end{array}$} & Moins de 30 ans & 0,27 & $0,04^{*}$ \\
\hline & $40-50$ ans & ns & 0,75 \\
\hline & 50 ans et plus & ns & $0,03 *$ \\
\hline \multirow{3}{*}{$\begin{array}{l}\text { Quartile de niveau de vie après la } \\
\text { séparation } \\
\text { Réf. : Quartile } 4\end{array}$} & Quartile 1 & 0,21 & $<0,001^{* *}$ \\
\hline & Quartile 2 & 0,47 & 0,07 \\
\hline & Quartile 3 & ns & 0,88 \\
\hline \multirow{3}{*}{$\begin{array}{l}\text { Catégorie socio-professionnelle par } \\
\text { rapport au conjoint } \\
\text { Réf.: Cadres, PI ou petits indépendants } \\
\text { ensemble }\end{array}$} & $\begin{array}{l}\text { Ouvriers'ères et Employées } \\
\text { ensemble }\end{array}$ & 0,41 & 0,05 \\
\hline & $\begin{array}{l}\text { Conjointe avec une PCS } \\
\text { inférieure }\end{array}$ & 0,37 & $0,02^{*}$ \\
\hline & $\begin{array}{l}\text { Conjointe avec une PCS } \\
\text { supérieure }\end{array}$ & 0,2 & $<0,0001^{* * *}$ \\
\hline $\begin{array}{l}\text { Type de logement après la séparation } \\
\text { Réf. : maison }\end{array}$ & Immeuble & 0,08 & $<0,0001^{* * *}$ \\
\hline \multirow{2}{*}{$\begin{array}{l}\text { Statut d'occupation avant la séparation } \\
\text { Réf : accédant à la propriété }\end{array}$} & Non propriétaire & 0,02 & $<0,0001^{* * *}$ \\
\hline & Propriétaire NA & ns & 0,16 \\
\hline \multirow{2}{*}{$\begin{array}{l}\text { Taille de l'U.U. après la séparation } \\
\text { Réf. : U.U. + } 100000 \text { habitants }\end{array}$} & $\begin{array}{l}\text { Unité urb. de moins de } 20000 \\
\text { hab. }\end{array}$ & 0,38 & $<0,001^{* *}$ \\
\hline & $\begin{array}{l}\text { Unité urb. de } 20000 \text { à } 100000 \\
\text { hab. }\end{array}$ & 0,17 & $<0,0001^{* * *}$ \\
\hline
\end{tabular}

Champ : les séparées $(\mathrm{N}=940)$

Note de lecture de l'odds ratio : en France, entre 2004 et 2015, les séparées en couple cohabitant ont 2,5 fois plus de chances d'être propriétaires que les célibataires, toutes choses égales par ailleurs

\section{Variables familiales et sexe}

Une régression supplémentaire, non présentée ici, confirme que les hommes, indépendamment de leurs caractéristiques familiales et professionnelles, ont plus de chances d'être propriétaires de leur logement que les femmes. La prise en compte conjointe du sexe et de la situation familiale permet ici de démontrer quel est l'effet de la présence d'enfants pour les hommes et les femmes sur la probabilité d'être propriétaire. 
Le contrôle par la situation conjugale après la séparation prend en compte les trajectoires de remise en couple, davantage associées à la propriété, sans que l'on connaisse leur origine.

Variables professionnelles après la séparation

Le niveau de diplôme, tout comme le fait d'avoir vécu une période de chômage après la séparation ou une période d'emploi précaire (CDD, emploi aidé, intérim), permettent une meilleure précision du modèle via le critère de minimisation de l'AIC, mais ils n'apparaissent pas dans le tableau car aucune de ces modalités n'est significative. Le quartile niveau de vie après la séparation a également été pris en compte tout comme l'âge au moment de la séparation.

Variables professionnelles avant la séparation

Des variables professionnelles avant la séparation, croisées avec les caractéristiques du conjoint, ont été intégrées au modèle (PCS comparée à celle du conjoint, situation d'emploi comparée à celle du conjoint). Les modalités de cette dernière variable, bien que participant à la puissance explicative du modèle, n'ont pas été reproduites parce qu'aucune n'est significative au seuil de $10 \%$. Les variables individuelles (PCS individuelle, situation d'emploi avant la séparation), n'ont pas été prises en compte. Multicolinéaires avec les variables croisées présentées plus haut, elles participent moins à la puissance explicative du modèle.

Variables logement et contrôles

La localisation du bien, le type de bien après la séparation et le statut d'occupation avant la séparation sont prises en compte. C'est le cas aussi de l'année d'enquête et de la situation d'hébergement.

Variables logement et contrôles

La localisation du bien, le type de bien après la séparation et le statut d'occupation avant la séparation sont prises en compte. C'est le cas aussi de l'année d'enquête et de la situation d'hébergement.

Variables logement et contrôles

La localisation du bien, le type de bien après la séparation et le statut d'occupation avant la séparation sont prises en compte. C'est le cas aussi de l'année d'enquête et de la situation d'hébergement.

\section{NOTES}

1. Si la propriété est totalement remboursée, l'un·e des ex-conjoint·es doit rembourser la moitié du logement conjugal à l'autre. Lorsque la propriété n'est pas totalement remboursée, celui ou celle qui reste propriétaire doit rembourser son ex-conjointe, et continuer seule le remboursement du crédit. Il ou elle peut aussi devenir propriétaire d'un autre bien.

2. Ce statut n'est pas en soi un statut plus valorisé que les autres mais dans le contexte institutionnel actuel, le fait de ne pas pouvoir y accéder constitue une inégalité.

3. Nous ne traitons, par défaut, que de la question des unions hétérosexuelles, les unions homosexuelles étant trop peu nombreuses pour avoir un poids statistique suffisant dans notre échantillon (Buisson et Lapinte, 2013). 
4. Du fait des effectifs annuels de séparées restreints nous n'avons pas pu repérer d'effet de la crise des subprimes dans nos données. Nous avons cependant contrôlé par l'année de séparation notre modèle de régression.

5. Les enquêtes longitudinales sont soumises au risque d'attrition. La situation de séparation conjugale, où les mobilités multiples sont fréquentes, est particulièrement propice à de tels biais. Nous avons néanmoins utilisé les pondérations longitudinales pour prendre en compte le plus possible ce risque d'attrition.

6. Dans l'enquête SRCV, les couples mariés, plus âgés en moyenne, sont davantage propriétaires que les couples non mariés. Néanmoins, c'est le processus de décohabitation et ses incidences sur la perte du statut de propriétaire qui nous intéresse ici, c'est pourquoi nous prenons en compte tous les types de désunions de cohabitant-es.

7. $28,5 \%$ sont locataires privés avant une séparation, $18,6 \%$ sont locataires sociaux et $4,9 \%$ sont logé'es gratuitement.

8. Contrairement à SRCV, ENL 2013 permet de connaître le nombre de déménagements. Il s'agit néanmoins d'une enquête transversale ne permettant pas de suivre les individus pendant trois ans comme le propose SRCV.

9. Les individus locataires en couple devenant propriétaires après une séparation sont rares : seuls $5 \%$ des séparées sont concernées. Ce sont plus souvent des hommes jeunes, diplômés, en couple dans des unités urbaines denses.

10. Les catégories socioprofessionnelles ont été recodées selon le schéma proposé par T. Amossé (2019).

11. L'hypergamie correspond à une situation de couple avec un conjoint d'une catégorie socioprofessionnelle supérieure.

12. Les travaux de C. Martin (1997) soulignent néanmoins la diversité des situations des ménages monoparentaux. Si la monoparentalité est liée à une baisse des ressources monétaires après les séparations conjugales, elle vient surtout renforcer des inégalités sociales déjà existantes.

13. Les propriétaires non endetté'es sont bien plus âgées. Environ un tiers (31,8\%) sont retraitées, contre $1,7 \%$ des propriétaires endettées.

14. En France, en 2012, la résidence alternée concerne $17 \%$ des décisions de justice relatives à l'établissement de la résidence de l'enfant après la séparation des parents. La résidence chez le père est fixée dans $12 \%$ des cas (Guillaunneau et Moreau, 2013).

15. Le niveau de vie correspond au revenu disponible (y compris les pensions alimentaires), divisé par le nombre d'unités de consommation, qui prend en compte la structure du ménage. La garde des enfants fait donc mécaniquement baisser le niveau de vie.

16. Il aurait été plus satisfaisant de présenter des proportions différenciées pour les hommes et les femmes. Néanmoins, les effectifs des modalités de chaque variable sont trop faibles pour que cela soit réalisable.

17. $31,9 \%$ des hommes ont quitté le statut de propriétaire. C'est le cas de 49,7 \% des femmes. La p-value est toujours inférieure à 0,05 , sauf pour les questions d'emploi où elle est inférieure à 0,1 . 18. Après la séparation, les cadres sont majoritairement propriétaires endettées dans des unités urbaines de plus 100000 habitants, alors que trois quarts des ouvrier'ères et employées sont propriétaires endettées dans des zones urbaines de moins de 20000 habitants (SRCV). Par ailleurs, les employées vivent plus souvent dans des unités urbaines denses que les ouvrierères, ce qui peut aussi expliquer ces différentes propensions à accéder à la propriété.

19. L'évolution du niveau de vie correspond au taux de croissance du niveau de vie, entre un an avant la séparation, et deux après, divisé en quartiles. La baisse du niveau de vie importante concerne le quart de la population dont le niveau de vie baisse d'au moins $26 \%$. La baisse du niveau de vie modérée concerne celles et ceux dont le niveau de vie a baissé de $25 \%$ à $4 \%$. Le troisième quart a vécu une stagnation ou petite (voire une légère ?) augmentation du niveau de vie (de $-4 \%$ à $+22 \%)$. Enfin, le dernier quart a vu son niveau de vie augmenter d'au moins $22 \%$. 
20. Cette population recoupe en partie la population des plus modestes après la séparation.

21. Les séparations avec enfant(s) à moins de 30 ans concernent les classes populaires, ce qui s'explique par l'âge moyen à la maternité plus précoce pour ces milieux. Les femmes les moins diplômées ont en 2012 leur premier enfant quatre ans plus tôt que les plus diplômées (Volant, 2017).

22. Il est de plus impossible de distinguer le mode de garde des enfants (garde simple ou garde alternée) avec SRCV : les situations de foyer monoparental recoupent donc ces deux catégories.

23. L'enquête SRCV ne permet pas de connaître l'âge des enfants hors domicile, ni les raisons pour lesquelles ils sont « hors domicile».

24. Il est impossible de distinguer le mode de garde des enfants (garde simple ou garde alternée) avec SRCV : les situations de foyer monoparental recoupent donc ces deux catégories.

25. Nous avons choisi cet intervalle de 25-50 ans pour comparer les personnes avec enfant(s) à celles n'en ayant pas, mais ayant une position similaire dans le cycle de vie.

26. La proportion de femmes dans cette situation est trop peu importante pour que les données soient utilisées.

27. Par exemple, les juges considèrent les crédits immobiliers des pères comme des dépenses incompressibles pour fixer les pensions alimentaires (Collectif Onze, 2013).

\section{RÉSUMÉS}

À partir de l'enquête SRCV (Statistiques sur les Ressources et les Conditions de Vie), cet article se propose de décrire comment les séparations conjugales viennent renforcer les inégalités d'accession à la propriété entre hommes et femmes. Avant la rupture, les femmes ont des carrières moins rémunératrices que leur conjoint. Ces inégalités se creusent après la séparation, d'autant plus que les femmes ont davantage la garde des enfants. Elles ont donc des niveaux de vie plus faibles et des situations professionnelles plus précaires, leur permettant moins souvent de rester propriétaires. Par ailleurs, à caractéristiques égales, les hommes font davantage le choix de la propriété que les femmes, notamment lorsqu'ils sont pères, venant confirmer que les « choix résidentiels » sont genrés.

How do break-ups reinforce inequalities in access to property between men and women? Using the French data of SILC (Statistics on Income and Living Conditions), we see that before the marital split, women already have less lucrative careers than their husbands. These inequalities are still there after the breakdown, especially since women get more child custody. They have lower standards of living and more precarious professional situations which makes it difficult to remain homeowners. On the other hand, with equal characteristics, men choose property more than women, especially when they are fathers. This confirms that "residential choices" are gendered.

\section{INDEX}

Mots-clés : France, accession à la propriété, inégalités de genre, séparations conjugales, trajectoires résidentielles

Keywords : France, home ownership, gender inequalities, marital breakups, residential pathways 
AUTEUR

LAURE CREPIN

Doctorante

Université Paris 8, CRESPPA-UMR 7217

laure.crepin01@gmail.com 